Presidential Address: Induction of the President, College of Pathologists of Sri Lanka 2019

\title{
Quality assurance in immunohistochemistry for better patient
}

\section{care}

Pathologists are traditionally considered diagnosticians. A histopathologist makes a diagnosis based on morphological features and provides information on the aetiology and prognosis of a lesion for management. This role has expanded over the decades especially with the advent of new biomarkers both immunohistochemical and molecular which have a major role to play in the era of Precision Medicine. The term 'Precision Medicine' replaces the term personalized medicine and refers to the tailoring of treatment to the individual characteristics of each patient by classifying them into subpopulations. This approach to medicine is emerging as the healthcare approach of tomorrow and have important implications for the entire healthcare system, including medical laboratories, expanding our landscape.

Acquiring sufficient breadth and depth of the exponential growth in new knowledge is a dire need for day to day histopathology practice. Every year, the College of Pathologists of Sri Lanka (CPSL) addresses this issue by organizing workshops and academic sessions. The biannual international conference organized in collaboration with the British Division of International Academy of Pathology (BDIAP) will be held in August 2019 , for the $7^{\text {th }}$ time and will be on updates in Lymphoid, Dermatopathology and Cytopathology.

With increase in the load and diversity of work, invariably the quality of work is at stake. We hope to address quality assurance in one of the most frequently needed ancillary test in histopathology, namely immunohistochemistry, as one of the main activities of the CPSL in 2019. Therefore, my main topic today is
"Quality assurance in immunohistochemistry for better patient care."

Immunohistochemistry has revolutionized the field of surgical pathology. It helps in refining the histopathological diagnosis, unraveling an unknown primary malignancy, detection of micro-invasive and micro-metastatic malignant disease and in predicting response to treatment. Immunohistochemistry, considered at its inception an ancillary technique, has now become a more standalone test especially for oncologists in the era of Precision Medicine. Estrogen and Progesterone receptors have been used as biomarkers for targeted endocrine therapy for breast cancer for more than three decades and it is the current standard of care. Trastuzumab therapy for HER2 positive breast cancers, Rituximab for $\mathrm{CD} 20$ expressing lymphomas and Imatinib for c-kit expressing gastrointestinal stromal tumours are few other examples. These biomarkers are detected on tissues using immunohistochemistry.

The Immunohistochemistry laboratory service was established in Sri Lanka, initially in 3 university histopathology laboratories: Colombo, Peradeniya and Ruhuna. The National Immunohistochemistry Laboratory Service was established in the year 2011 at the National Hospital of Sri Lanka. This service has now been expanded and immunohistochemistry facility is available at Teaching Hospitals Kandy, Galle, Anuradhapura and Jaffna, Apeksha Hospital Maharagama and National Hospital for Respiratory Diseases. It will be extended further to more laboratories in the near future (Figure1). 
Over the past 25 years, in many countries, great efforts have been made to standardize diagnostic immunohistochemistry results especially with the establishment of external quality control for immunohistochemistry. Advances in technology has resulted in improved quality of antibodies, automated platforms and highly sensitive detection systems improving the reproducibility and reliability of IHC results. Currently, the immunohistochemistry laboratories of the Ministry of Health, use automated platforms and highly sensitive detection systems. However, none of these advances replaces the need for external quality assurance.

As with any test, laboratories must ensure that $\mathrm{IHC}$ test results are accurate and reproducible and that the test performs as planned. All IHC laboratories in the country use positive controls as the internal quality control measure, where a tissue known to express the marker is included with the staining of tissue under test. If the positive control becomes positive, we assume that the test has worked correctly and the results are acceptable.

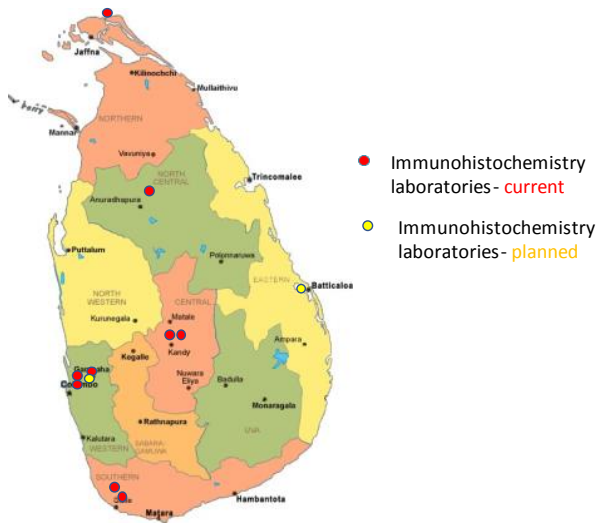

Figure 1: Distribution of Immunohistochemistry laboratory services in Sri Lanka

Immunohistochemistry is a complex procedure and the results depend on many factors. Proper tissue fixation and processing are preanalytical steps that can influence the result. Antibody selection and optimization are the main analytical factors affecting the results. Correct use of controls and correct interpretation of the stained slides by the pathologist, are the important post analytical factors, which has to be brought under control to ensure the accuracy of the results.

Standardization of every step in the preanalytical, analytical and post-analytical phases is crucial to achieve reproducible and reliable immunohistochemistry test results. Reproducibility is the ability of a test to produce the same result in repeat testing. Reproducibility of a qualitative test like immunohistochemistry is similar to precision of a quantitative test. Staining quality can vary greatly among different laboratories, depending on the technical expertise and protocols used. The final interpretation of the slide can also vary between pathologists, affecting the reliability and reproducibility of the test.

Although we use positive controls to assure the reproducibility of results of individual laboratories, it will not necessarily identify poorly calibrated IHC detection systems giving insufficient staining. In contrast, external quality assessment enables the identification of insufficient staining and interpretation problems as well. The major benefits of an external quality assurance programme cannot be achieved by internal quality control alone. EQA programmes give early warnings to problems with antibodies and provide objective evidence of the quality of the immunohistochemistry laboratory, guiding the lab as to where in the process the improvement needs to be made.

Therefore, the Council of the CPSL decided to design an external quality assurance programme for immunohistochemistry, to improve the accuracy of test results and to ensure that patients receive optimum care. It was designed to be implemented in 3 phases. In phase 1 the IHC EQA programme was designed and implemented as a pilot project last year. For phase 2 an educational programme addressing the deficiencies will be held in 2019. Phase 3 is to implement the programme as a national project.

As prognostic immunohistochemical markers of breast cancer takes a major part of the 
workload of immunohistochemistry laboratories, the pilot project was designed to assess the inter laboratory and inter-observer reliability in reporting ER, PR and Her2, the predictive markers of breast cancer.

I would like to share with you some of the findings of our pilot project.

- The interobserver reliability of reporting whether a breast cancer is positive or negative for ER was commendable as it was perfect in $42 \%$ of laboratories. The Histopathologists have obtained the same results by reporting on the EQA slides stained in their laboratories

- Other laboratories had a good to moderate reliability indicating that there is need for improvement in reporting ER status at $58 \%$ of laboratories.

- Only $40 \%$ of laboratories had excellent to good inter-observer reliability while the others had only moderate interobserver reliability in identifying positive and negative expression of $P R$ and Her2 indicating need for further improvement.

- When the inter laboratory reliability in producing correct staining to identify positive ER expression was assessed, it was found that there was excellent reliability between the laboratories.

- However, only a moderate reliability was found in obtaining correct staining for PR and Her 2.

- Technical aspects of PR and Her2 need to be addressed.

I would like to illustrate two examples.

These two pictures (Figure2) show immunostaining obtained in two laboratories for ER expression done on the same tissue block. The picture A shows low but specific nuclear expression of ER which would be reported positive, according to the guidelines. The picture $B$ shows completely negative expression of ER.

What is the implication?

According to the report issued from Lab A, the patient will receive hormone therapy for breast cancer while the report issued by Lab B will deprive the patient of receiving targeted therapy.

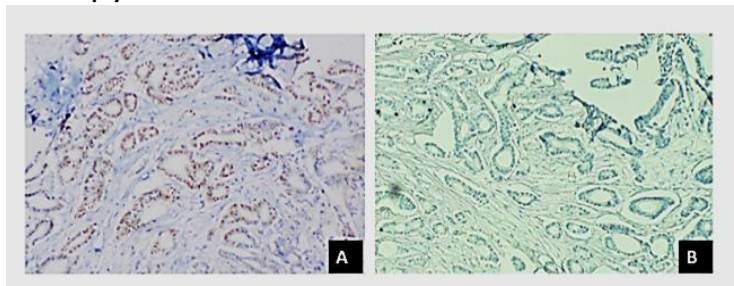

Figure 2: Variation in IHC staining in low ER expressing breast cancer

This is another example (Figure 3). These are pictures of immunohistochemistry for Her2 expression in a single tissue block done at 5 laboratories. According to the Lab E, Her 2 is negative. Lab B, C and D should go for FISH for confirmation as they are showing equivocal staining for Her 2. According to the staining in Lab A, a histopathologist who is unaware of the staining quality of laboratory $A$ may report the test as positive. But it should be repeated as there is unacceptable background staining precluding the assessment.

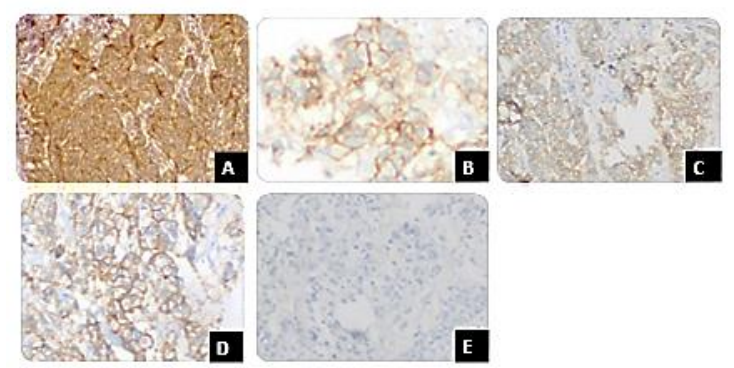

Figure 3: Variation in IHC staining in low Her2 expressing breast cancer

What are the reasons for such variation which prevents excellent to perfect reliability?

Out of the 3 phases of immunohistochemical testing, pre-analytical variables are considered the 'weakest link' of immunohistochemistry. Poorly fixed tissue compromises the accuracy of $\mathrm{IHC}$ results. Reproducibility of staining between laboratories also becomes difficult. Simply said, if you put garbage in, you get garbage out. It is hard to standardize the pre analytical stage as they are often out of control of the pathologist. Therefore, very often we need to optimize our staining protocols to get maximum validity of the results, which requires technical expertise. 
One other important observation made in this pilot study was that the positive controls used, are not optimal to identify the low level of expression of the biomarker (Figure 4). All laboratories use high expression positive controls. The cut off value which determines the clinically important expression level for ER and PR are low and may be missed due to under staining if we do not use low expression controls.

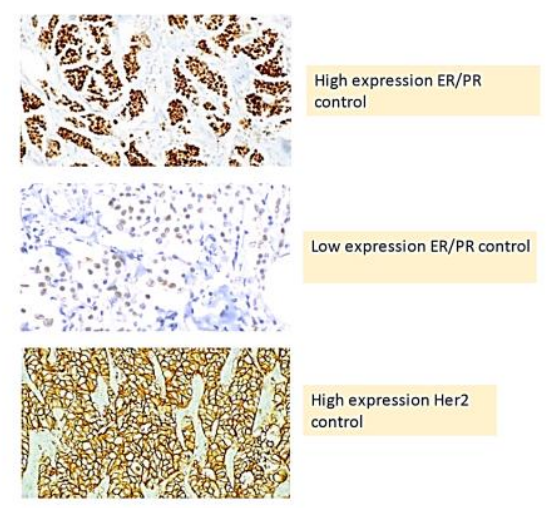

Figure 4: Types of controls used for ER/PR and Her2

Failure to include a low expression control leads to increase in false negative rates, depriving the patient from receiving targeted therapy. This issue had been addressed very recently and the concept of critical assay performance controls (CAPCs) has been introduced to overcome this problem in some countries.

Quality assurance in immunohistochemistry is still evolving parallel to the development of more refined detection systems and automated platforms. Pathologists as well as technical personnel attending to the assays need to acquire the rapidly advancing knowledge in immunohistochemistry. The CPSL is planning to conduct a series of workshops to keep abreast of these advances and quality assurance in immunohistochemistry.

The results of this pilot study emphasize the need for implementation of an immunohistochemistry external quality assurance programme for Sri Lanka. Although immunohistochemistry has been an important tool for histopathologists and has been in use for more than a decade in our country, its technical aspects and quality control took long years to get the spotlight it deserves. We have taken up the challenge of assuring the quality of immunohistochemistry tests and earnestly request the support of the Ministry of Health, the apex body which looks after health care of the country, to make EQA for IHC an inbuilt facility.

The pilot project on EQA in IHC was completed with the dedication of the members of our college.

I have discussed a single challenge we face in the expanded landscape.

As the key professional body in the field of pathology in Sri Lanka, CPSL should strive hard to meet the challenges of the expanding role of the pathologist. This is not a task which can be completed within a year or two. Challenges would continue to surface and we need to work together to reshape our role to accommodate the advances in the field, at the same time addressing the ever emerging challenges that are linked with such advances.

\section{Prof. Lakmini Mudduwa}

President, College of Pathologists of Sri Lanka 2019

Senior Professor of Pathology

Department of Pathology

Faculty of Medicine, University of Ruhuna,

Galle, Sri Lanka

$8^{\text {th }}$ February 2019

Hotel Ramada, Colombo, Sri Lanka 